\title{
On short zero-sum subsequences of zero-sum sequences
}

\author{
Yushuang Fan \\ Center for Combinatorics \\ Nankai University, LPMC-TJKLC \\ Tianjin, P. R. China \\ fys850820@163.com \\ Guoqing Wang* \\ Department of Mathematics \\ Tianjin Polytechnic University \\ Tianjin, P. R. China \\ gqwang1979@yahoo.com.cn
}

\author{
Weidong Gao \\ Center for Combinatorics \\ Nankai University, LPMC-TJKLC \\ Tianjin, P. R. China \\ wdgao1963@yahoo.com.cn \\ Qinghai Zhong \\ Center for Combinatorics \\ Nankai University, LPMC-TJKLC \\ Tianjin, P. R. China \\ zhongqinghai@yahoo.com.cn
}

\author{
Department of Mathematics \\ Dalian Maritime University \\ Dalian, P. R. China \\ jjzhuang1979@yahoo.com.cn
}

Submitted: Aug 14, 2011; Accepted: Aug 23, 2012; Published: Sep 6, 2012

Mathematics Subject Classifications: 11B75, 11P99

\begin{abstract}
Let $G$ be a finite abelian group of exponent $\exp (G)$. By $D(G)$ we denote the smallest integer $d \in \mathbb{N}$ such that every sequence over $G$ of length at least $d$ contains a nonempty zero-sum subsequence. By $\eta(G)$ we denote the smallest integer $d \in \mathbb{N}$ such that every sequence over $G$ of length at least $d$ contains a zero-sum subsequence $T$ with length $|T| \in[1, \exp (G)]$, such a sequence $T$ will be called a short zero-sum sequence. Let $C_{0}(G)$ denote the set consists of all integer $t \in[D(G)+1, \eta(G)-1]$ such that every zero-sum sequence of length exactly $t$ contains a short zero-sum subsequence. In this paper, we investigate the question whether $C_{0}(G) \neq \emptyset$ for all non-cyclic finite abelian groups $G$. Previous results showed that $C_{0}(G) \neq \emptyset$ for the groups $C_{n}^{2}(n \geqslant 3)$ and $C_{3}^{3}$. We show that more groups including the groups $C_{m} \oplus C_{n}$ with $3 \leqslant m \mid n, C_{3^{a} 5^{b}}^{3}, C_{3 \times 2^{a}}^{3}, C_{3^{a}}^{4}$ and $C_{2^{b}}^{r}(b \geqslant 2)$ have this property. We
\end{abstract}

*Corresponding author: Guoqing Wang 
also determine $C_{0}(G)$ completely for some groups including the groups of rank two, and some special groups with large exponent.

Keywords: Zero-sum sequence; short zero-sum sequence; short free sequence; zerosum short free sequence; Davenport constant

\section{Introduction}

Let $G$ be an additive finite abelian group of exponent $\exp (G)$. We call a zero-sum sequence $S$ over $G$ a short zero-sum sequence if $1 \leqslant|S| \leqslant \exp (G)$. Let $\eta(G)$ be the smallest integer $d$ such that every sequence $S$ over $G$ of length $|S| \geqslant d$ contains a short zero-sum subsequence. Let $D(G)$ be the Davenport constant of $G$, i.e., the smallest integer $d$ such that every sequence over $G$ of length at least $d$ contains a nonempty zero-sum subsequence. Both $D(G)$ and $\eta(G)$ are classical invariants in combinatorial number theory. For detail on terminology and notation we refer to Section 2.

By the definition of $\eta(G)$ we know that for every integer $t \in[1, \eta(G)-1]$, there is a sequence $S$ over $G$ of length exactly $t$ such that $S$ contains no short zero-sum subsequence. In this paper, we consider the following problem related to $D(G)$ and $\eta(G)$, which was first investigated by Emde Boas in the late sixties. Given a finite abelian group, what are integers $\exp (G)+1 \leqslant t \leqslant \eta(G)-1$, if any, such that every zero-sum sequence $S$ over $G$ of length $|S|=t$ contains a short zero-sum subsequence. Denote by $C_{0}(G)$ the set of all those integers $t$. It will be readily seen that $C_{0}(G) \subset[D(G)+1, \eta(G)-1]$.

In 1969, Emde Boas and D. Kruyswijk [7] proved that $14 \in C_{0}\left(C_{3}^{3}\right)$. In 1997, the second author of this paper showed that $[2 q, 3 q-3] \subset C_{0}\left(C_{q}^{2}\right)$, where $q$ is a prime power.

Let us first make some easy observations on $C_{0}(G)$. Note that for every $t \in[1, D(G)]$ there exists a minimal zero-sum sequence over $G$ of length $t$. So, $C_{0}(G) \subset[D(G)+$ 1, $\eta(G)-1]$ follows from the easy fact that $D(G) \geqslant \exp (G)$.

If $G=C_{2} \oplus C_{2 m}$ then $D(G)+1=2 m+2$ and $\eta(G)-1=2 m+1$. Therefore, by the definition we have $C_{0}\left(C_{2} \oplus C_{2 m}\right)=\emptyset$. We suggest the following

Conjecture 1. Let $G$ be a non-cyclic finite abelian group. If $G \neq C_{2} \oplus C_{2 m}$ then $C_{0}(G) \neq$ $\emptyset$.

In this paper we shall determine $C_{0}(G)$ completely for some groups.

Theorem 2. Let $G$ be a non-cyclic finite abelian group, and let $r(G)$ be the rank of $G$. Then,

1. $C_{0}(G)=[D(G)+1, \eta(G)-1]$ if $r(G)=2$.

2. $C_{0}(G)=[D(G)+1, \eta(G)-1]$ if $G=C_{m p^{n}} \oplus H$ with $p$ a prime, $H$ a p-group and $p^{n} \geqslant D(H)$.

3. $C_{0}\left(C_{3}^{4}\right)=\left\{\eta\left(C_{3}^{4}\right)-2, \eta\left(C_{3}^{4}\right)-1\right\}=\{37,38\}$. 
4. $C_{0}\left(C_{2}^{r}\right)=\left\{\eta\left(C_{2}^{r}\right)-3, \eta\left(C_{2}^{r}\right)-2\right\}$, where $r \geqslant 3$.

We also confirm Conjecture 1 for more groups other than those listed in Theorem 2.

Theorem 3. If $G$ is one of the following groups then $C_{0}(G) \neq \emptyset$.

1. $G=C_{3^{a} 5^{b}}^{3}$ where $a \geqslant 1$ or $b \geqslant 2$.

2. $G=C_{3 \times 2^{a}}^{3}$ where $a \geqslant 4$.

3. $G=C_{3^{a}}^{4}$ where $a \geqslant 1$.

4. $G=C_{2^{a}}^{r}$ where $3 \leqslant r \leqslant a$, or $a=1$ and $r \geqslant 3$.

5. $G=C_{k}^{3}$ where $k=3^{n_{1}} 5^{n_{2}} 7^{n_{3}} 11^{n_{4}} 13^{n_{5}}, n_{1} \geqslant 1, n_{3}+n_{4}+n_{5} \geqslant 3$, and $n_{1}+n_{2} \geqslant$ $11+34\left(n_{3}+n_{4}+n_{5}\right)$.

The rest of this paper is organized as follows: In Section 2 we introduce some notations and some preliminary results; In Section 3 we prove three lemmas connecting $C_{0}(G)$ with property C; In Section 4 we shall derive some lower bounds on $\min \left\{C_{0}(G)\right\}$; In Section 5 we study $C_{0}(G)$ with focus on the groups $C_{3}^{r}$; In Section 6 and 7 we shall prove Theorem 2 and Theorem 3, respectively; and in the final Section 8 we give some concluding remarks and some open problems.

\section{$2 \quad$ Notations and some preliminary results}

Our notations and terminologies are consistent with [10] and [13]. We briefly gather some key notions and fix the notations concerning sequences over finite abelian groups. Let $\mathbb{Z}$ denote the set of integers. Let $\mathbb{N}$ denote the set of positive integers, and $\mathbb{N}_{0}=\mathbb{N} \cup\{0\}$. For real numbers $a, b \in \mathbb{R}$, we set $[a, b]=\{x \in \mathbb{Z}: a \leqslant x \leqslant b\}$. Throughout this paper, all abelian groups will be written additively, and for $n, r \in \mathbb{N}$, we denote by $C_{n}$ a cyclic group with $n$ elements, and denote by $C_{n}^{r}$ the direct sum of $r$ copies of $C_{n}$.

Let $G$ be a finite abelian group and $\exp (G)$ its exponent. By $r(G)$ we denote the rank of $G$. A sequence $S$ over $G$ will be written in the form

$$
S=g_{1} \cdot \ldots \cdot g_{\ell}=\prod_{g \in G} g^{v_{g}(S)}, \quad \text { with } \vee_{g}(S) \in \mathbb{N}_{0} \text { for all } g \in G,
$$

and we call

$$
|S|=\ell \in \mathbb{N}_{0} \quad \text { the length and } \quad \sigma(S)=\sum_{i=1}^{\ell} g_{i}=\sum_{g \in G} \mathrm{v}_{g}(S) g \in G \quad \text { the sum of } S .
$$

Let $\operatorname{supp}(S)=\left\{g \in G: \mathrm{v}_{g}(S)>0\right\}$. We call $S$ a square free sequence if $\mathrm{v}_{g}(S) \leqslant 1$ for every $g \in G$. So, a square free sequence over $G$ is actually a subset of $G$. A sequence $T$ 
over $G$ is called a subsequence of $S$ if $v_{g}(T) \leqslant v_{g}(S)$ for every $g \in G$, and denote by $T \mid S$. For every $r \in[1, \ell]$, define

$$
\sum_{\leqslant r}(S)=\{\sigma(T): T|S, 1 \leqslant| T \mid \leqslant r\}
$$

and define

$$
\sum(S)=\{\sigma(T): T|S,| T \mid \geqslant 1\} .
$$

The sequence $S$ is called

- a zero-sum sequence if $\sigma(S)=0$.

- a short zero-sum sequence over $G$ if it is a zero-sum sequence of length $|S| \in$ $[1, \exp (G)]$.

- a short free sequence over $G$ if $S$ contains no short zero-sum subsequence.

So, a zero-sum sequence over $G$ which contains no short zero-sum subsequence will be called a zero-sum short free sequence over $G$.

For every element $g \in G$, we set $g+S=\left(g+g_{1}\right) \cdot \ldots \cdot\left(g+g_{\ell}\right)$. Every map of abelian groups $\varphi: G \rightarrow H$ extents to a map from the sequences over $G$ to the sequences over $H$ by $\varphi(S)=\varphi\left(g_{1}\right) \cdot \ldots \cdot \varphi\left(g_{\ell}\right)$. If $\varphi$ is a homomorphism, then $\varphi(S)$ is a zero-sum sequence if and only if $\sigma(S) \in \operatorname{ker}(\varphi)$.

In the rest of this section we gather some known results which will be used in the sequel.

We shall study $C_{0}(G)$ by using the following property which was first introduced and investigated by Emde Boas and Kruyswijk [7] in 1969 for the groups $C_{p}^{2}$ with $p$ a prime, and was investigated in 2007 for the groups $C_{n}^{r}$ by the second author, Geroldinger and Schmid [12].

Property C: We say the group $C_{n}^{r}$ has property $C$ if $\eta\left(C_{n}^{r}\right)=c(n-1)+1$ for some positive integer $c$, and every short free sequence $S$ over $C_{n}^{r}$ of length $|S|=c(n-1)$ has the form $S=\prod_{i=1}^{c} g_{i}^{n-1}$ where $g_{1}, \ldots, g_{c}$ are pairwise distinct elements of $C_{n}^{r}$.

It is conjectured that every group of the form $C_{n}^{r}$ has Property C(see [10], Section 7).

We need the following result which states that Property $\mathrm{C}$ is multiple.

Lemma 4. ([12], Theorem 3.2) Let $G=C_{m n}^{r}$ with $m, n, r \in \mathbb{N}$. If both $C_{m}^{r}$ and $C_{n}^{r}$ have Property $C$ and

$$
\frac{\eta\left(C_{m}^{r}\right)-1}{m-1}=\frac{\eta\left(C_{n}^{r}\right)-1}{n-1}=\frac{\eta\left(C_{m n}^{r}\right)-1}{m n-1}=c
$$

for some $c \in \mathbb{N}$ then $G$ has Property $C$.

We also need the following old easy result. 
Lemma 5. ([20]) $D\left(C_{n}^{3}\right) \geqslant 3 n-2$.

Definition 6. Let $G$ be a finite abelian group. Define $g(G)$ to be the smallest integer $t$ such that every square free sequence over $G$ of length $t$ contains a zero-sum subsequence of length $\exp (G)$. Let $f(G)$ be the smallest integer $t$ such that every square free sequence over $G$ of length $t$ contains a short zero-sum subsequence.

We now gather some known results on Property C, $\eta(G), g(G)$ and $f(G)$ which will be used in the sequel.

Lemma 7. Let $r, t \in \mathbb{N}$, and let $n \geqslant 3$ be an odd integer. Then,

1. $\eta\left(C_{n}^{3}\right) \geqslant 8 n-7$. ([6], or [5], Theorem 1.2)

2. $\eta\left(C_{n}^{4}\right) \geqslant 19 n-18$. ([5], Theorem 1.3)

3. $\eta\left(C_{3}^{3}\right)=17$. ([19], or [6], page 3)

4. $\eta\left(C_{3}^{4}\right)=39$ and $g\left(C_{3}^{4}\right)=21$. ([19], or [6], page 3)

5. $\eta\left(C_{5}^{3}\right)=33=8 \times 5-7$. ([11], Theorem 1.7)

6. $\eta\left(C_{2^{t}}^{r}\right)=\left(2^{r}-1\right)\left(2^{t}-1\right)+1$. ([18])

7. $\eta\left(C_{3 \times 2^{\alpha}}^{3}\right)=7\left(3 \times 2^{\alpha}-1\right)+1$ where $\alpha \geqslant 1$. ([11], Theorem 1.8)

8. $C_{5}^{3}$ has Property C. ([11], Theorem 1.9)

9. $\eta\left(C_{3}^{r}\right)=2 f\left(C_{3}^{r}\right)-1$. ([18])

10. $C_{3}^{r}$ has Property C. ([18])

Lemma 8. ([5], Lemma 5.4) Let $r \in[3,5]$, and let $S$ and $S^{\prime}$ be two square free sequences over $C_{3}^{r}$ of length $|S|=\left|S^{\prime}\right|=g\left(C_{3}^{r}\right)-1$. Suppose that both $S$ and $S^{\prime}$ contain no zero-sum subsequence of length 3 . Then $S^{\prime}=\varphi(S)+a$, where $\varphi$ is an automorphism of $C_{3}^{r}$ and $a \in C_{3}^{r}$.

Lemma 9. ([1], Lemma 1) Let $T$ be a square free sequence over $C_{3}^{3}$ of length 8. If $T$ contains no short zero-sum subsequence then there exists an automorphism $\varphi$ of $C_{3}^{3}$ such that

$$
\varphi(T)=\left(\begin{array}{l}
0 \\
1 \\
0
\end{array}\right)\left(\begin{array}{l}
0 \\
0 \\
1
\end{array}\right)\left(\begin{array}{l}
0 \\
1 \\
1
\end{array}\right)\left(\begin{array}{l}
1 \\
0 \\
0
\end{array}\right)\left(\begin{array}{l}
1 \\
2 \\
0
\end{array}\right)\left(\begin{array}{l}
1 \\
1 \\
1
\end{array}\right)\left(\begin{array}{l}
1 \\
1 \\
2
\end{array}\right)\left(\begin{array}{l}
2 \\
0 \\
1
\end{array}\right) .
$$


Lemma 10. ([3]; [5], page 182) The following square free sequence over $C_{3}^{4}$ of length 20 contains no zero-sum subsequence of length 3.

$$
\begin{aligned}
& \left(\begin{array}{l}
0 \\
0 \\
0 \\
0
\end{array}\right)\left(\begin{array}{l}
2 \\
0 \\
0 \\
0
\end{array}\right)\left(\begin{array}{l}
0 \\
2 \\
0 \\
0
\end{array}\right)\left(\begin{array}{l}
2 \\
2 \\
0 \\
0
\end{array}\right)\left(\begin{array}{l}
1 \\
0 \\
2 \\
0
\end{array}\right)\left(\begin{array}{l}
0 \\
1 \\
2 \\
0
\end{array}\right)\left(\begin{array}{l}
1 \\
2 \\
2 \\
0
\end{array}\right)\left(\begin{array}{l}
2 \\
1 \\
2 \\
0
\end{array}\right)\left(\begin{array}{l}
1 \\
1 \\
1 \\
0
\end{array}\right)\left(\begin{array}{l}
1 \\
1 \\
0 \\
1
\end{array}\right) \\
& \left(\begin{array}{l}
0 \\
0 \\
2 \\
2
\end{array}\right)\left(\begin{array}{l}
2 \\
0 \\
2 \\
2
\end{array}\right)\left(\begin{array}{l}
0 \\
2 \\
2 \\
2
\end{array}\right)\left(\begin{array}{l}
2 \\
2 \\
2 \\
2
\end{array}\right)\left(\begin{array}{l}
1 \\
0 \\
0 \\
2
\end{array}\right)\left(\begin{array}{l}
0 \\
1 \\
0 \\
2
\end{array}\right)\left(\begin{array}{l}
1 \\
2 \\
0 \\
2
\end{array}\right)\left(\begin{array}{l}
2 \\
1 \\
0 \\
2
\end{array}\right)\left(\begin{array}{l}
1 \\
1 \\
1 \\
2
\end{array}\right)\left(\begin{array}{l}
1 \\
1 \\
2 \\
1
\end{array}\right) .
\end{aligned}
$$

Lemma 11. ([15], Theorem 5.2) Every sequence $S$ over $C_{n}^{2}$ of length $|S|=3 n-2$ contains a zero-sum subsequence of length $n$ or $2 n$.

Lemma 12. ([12], Lemma 4.5) Let $G$ be a finite abelian group, and let $H$ be a proper subgroup of $G$ with $\exp (G)=\exp (H) \exp (G / H)$. Then $\eta(G) \leqslant(\eta(H)-1) \exp (G / H)+$ $\eta(G / H)$.

Lemma 13. Let $p$ be a prime and let $H$ be a finite abelian $p$-group such that $p^{n} \geqslant D(H)$. Let $n_{1}, n_{2}, m, n \in \mathbb{N}$ with $n_{1} \mid n_{2}$. Then,

1. $D\left(C_{n_{1}} \oplus C_{n_{2}}\right)=n_{1}+n_{2}-1$. ([20])

2. $D\left(C_{m p^{n}} \oplus H\right)=m p^{n}+D(H)-1$. ([r]

3. Let $G=C_{p^{e_{1}}} \oplus \cdots \oplus C_{p^{e_{r}}}$ with $e_{i} \in \mathbb{N}$. Then, $D(G)=1+\sum_{i=1}^{r}\left(p^{e_{i}}-1\right)$. ([20])

4. $\eta\left(C_{n_{1}} \oplus C_{n_{2}}\right)=2 n_{1}+n_{2}-2$. ([14])

5. Let $G=H \oplus C_{n}$ with $\exp (H) \mid n \geqslant 2$. Then, $\eta(G) \geqslant 2(D(H)-1)+n$. ([5])

We also need the following easy lemma

Lemma 14. ([16] Lemma 4.2.2) Let $G$ be a finite abelian group. Then, $\mathrm{s}(G) \geqslant \eta(G)+$ $\exp (G)-1$.

We shall show that the following property can also be used to study $C_{0}(G)$.

Property $D_{0}:$ Let $c, n \in \mathbb{N}$. We say that $C_{n}^{r}$ has property $D_{0}$ with respect to $c$ if every sequence of the form $g \prod_{i=1}^{c} g_{i}^{n-1}$ contains a zero-sum subsequence of length exactly $n$, where $g, g_{i} \in C_{n}^{r}$ for all $i \in[1, c]$.

Lemma 15. ([8], page 8) Let $m=3^{a} 5^{b}$ with a,b nonnegative integers. Let $n \geqslant 65$ be an odd positive integer such that $C_{p}^{3}$ has Property $D_{0}$ with respect to 9 for all prime divisors $p$ of $n$. If

$$
m \geqslant \frac{2 \times 5^{7} n^{17}}{\left(n^{2}-7\right) n-64}
$$

then $\mathrm{s}\left(C_{m n}^{3}\right)=9 m n-8$. 


\section{Three lemmas connecting $C_{0}(G)$ with Property C}

Lemma 16. Let $G=C_{n}^{r}$ with $\eta(G)=c(n-1)+1$ for some $c \in \mathbb{N}$. If $c \leqslant n$ and if $G$ has Property $C$ then $\eta(G)-1 \in C_{0}(G)$.

Proof. Let $S$ be a zero-sum sequence over $G$ of length $|S|=\eta(G)-1=c(n-1)$. We need to show that $S$ contains a short zero-sum subsequence. If $S=\prod_{i=1}^{c} g_{i}^{n-1}$ for some $g_{i} \in G$, then $(n-1)\left(g_{1}+g_{2}+\cdots+g_{c}\right)=\sigma(S)=0=n\left(g_{1}+g_{2}+\cdots+g_{c}\right)$. It follows that $g_{1}+g_{2}+\cdots+g_{c}=0$. Therefore, $g_{1} g_{2} \cdot \ldots \cdot g_{c}$ is a zero-sum subsequence of $S$ of length $c \leqslant n$ and we are done. Otherwise, $S \neq \prod_{i=1}^{c} g_{i}^{n-1}$ for any $g_{i} \in G$. It follows from $G$ having Property $C$ that $S$ contains a short zero-sum subsequence.

Lemma 17. Let $G$ be a finite abelian group, and let $H$ be a proper subgroup of $G$ with $\exp (G)=\exp (H) \exp (G / H)$. Suppose that the following conditions hold.

(i) $\eta(G)=(\eta(H)-1) \exp (G / H)+\eta(G / H)$;

(ii) $G / H \cong C_{n}^{r}$ has Property $C$;

(iii) There exist $t_{1} \in[1, \exp (G / H)-1]$ and $t_{2} \in\{1,2\}$ such that $t_{2} \leqslant t_{1}$ and such that $\left[\eta(G / H)-t_{1}, \eta(G / H)-t_{2}\right] \subset C_{0}(G / H)$.

Then,

$$
\left[\eta(G)-t_{1}, \eta(G)-t_{2}\right] \subset C_{0}(G) .
$$

Proof. To prove this lemma, we assume to the contrary that there is a zero-sum short free sequence $S$ over $G$ of length $\eta(G)-t$ for some $t \in\left[t_{2}, t_{1}\right]$. Let $\varphi$ be the natural homomorphism from $G$ onto $G / H$.

Note that

$$
|S|=\eta(G)-t=(\eta(H)-1) \exp (G / H)+(\eta(G / H)-t) .
$$

This allows us to take an arbitrary decomposition of $S$

$$
S=\left(\prod_{i=1}^{\eta(H)-1} S_{i}\right) \cdot S^{\prime}
$$

with

$$
\left|S_{i}\right| \in[1, \exp (G / H)]
$$

and

$$
\sigma\left(S_{i}\right) \in \operatorname{ker}(\varphi)=H
$$

for every $i \in[1, \eta(H)-1]$.

Combining (3.1), (3.2), (3.3) and (3.4) we infer that

$$
\left|S^{\prime}\right| \geqslant \eta(G / H)-t \geqslant \eta(G / H)-t_{1}
$$

and

$$
\sigma\left(\varphi\left(S^{\prime}\right)\right)=0
$$


Claim. $\varphi\left(S^{\prime}\right)$ contains no zero-sum subsequence of length in $[1, \exp (G / H)]$. Proof of the claim. Assume to the contrary that, there exists a subsequence $S_{\eta(H)}$ (say) of $S^{\prime}$ of length $\left|S_{\eta(H)}\right| \in[1, \exp (G / H)]$ such that $\sigma\left(S_{\eta(H)}\right) \in \operatorname{ker}(\varphi)=H$. It follows that the sequence $U=\prod_{i=1}^{\eta(H)} \sigma\left(S_{i}\right)$ contains a zero-sum subsequence $W=\prod_{i \in I} \sigma\left(S_{i}\right)$ over $H$ with $I \subset[1, \eta(H)]$ and $|W|=|I| \in[1, \exp (H)]$. Therefore, the sequence $\prod_{i \in I} S_{i}$ is a zero-sum subsequence of $S$ over $G$ with $1 \leqslant\left|\prod_{i \in I} S_{i}\right| \leqslant|I| \exp (G / H) \leqslant \exp (H) \exp (G / H)=$ $\exp (G)$, a contradiction. This proves the claim.

By (3.5), (3.6), the above claim and Condition (iii), we conclude that

$$
t_{2}=2
$$

and

$$
\left|S^{\prime}\right|=\eta(G / H)-1 .
$$

This together with Condition (ii) implies that

$$
\varphi\left(S^{\prime}\right)=x_{1}^{n-1} \cdot \ldots \cdot x_{c}^{n-1}
$$

where $c=\frac{\eta(G / H)-1}{n-1}$ and $x_{1}, \ldots, x_{c}$ are pairwise distinct elements of the quotient group $G / H$. So, we just proved that every decomposition of $S$ satisfying conditions (3.3) and (3.4) has the properties (3.5)-(3.8).

Since $t \leqslant t_{1} \leqslant \exp (G / H)-1$, it follows from (3.1), (3.3) and (3.7) that $\left|S_{i}\right| \in$ $[2, \exp (G / H)]$ for all $i \in[1, \eta(H)-1]$. Moreover, since $t \geqslant t_{2}=2$, it follows that there exists $j \in[1, \eta(H)-1]$ such that $\left|S_{j}\right| \leqslant \exp (G / H)-1$. Without loss of generality we assume that

$$
\left|S_{1}\right| \in[2, \exp (G / H)-1] .
$$

Suppose that there exists $h \in \operatorname{supp}\left(\varphi\left(S_{1}\right)\right) \cap \operatorname{supp}\left(\varphi\left(S^{\prime}\right)\right)$. By (3.8), we have that the sequence $S_{1} \cdot S^{\prime}$ contains a subsequence $S_{1}^{\prime}$ with $\varphi\left(S_{1}^{\prime}\right)=h^{n}$. Let $S^{\prime \prime}=S_{1} \cdot S^{\prime} \cdot S_{1}^{\prime-1}$. We get a decomposition $S=S_{1}^{\prime} \cdot\left(\prod_{i=2}^{\eta(H)-1} S_{i}\right) \cdot S^{\prime \prime}$ satisfying (3.3) and (3.4). But $\left|S^{\prime \prime}\right|=$ $\left|S_{1}\right|+\left|S^{\prime}\right|-\left|S_{1}^{\prime}\right| \leqslant(n-1)+(\eta(G / H)-1)-n=\eta(G / H)-2$, a contradiction on (3.7). Therefore,

$$
\operatorname{supp}\left(\varphi\left(S_{1}\right)\right) \cap \operatorname{supp}\left(\varphi\left(S^{\prime}\right)\right)=\emptyset .
$$

Take a term $g \mid S_{1}$. Since $\varphi(g) \notin \operatorname{supp}\left(\varphi\left(S^{\prime}\right)\right)$ and $\left|S^{\prime} \cdot g\right|=\eta(G / H)$, it follows from the above claim that $S^{\prime} \cdot g$ contains a subsequence $S_{1}^{\prime}$ with

$$
g \mid S_{1}^{\prime}
$$

and

$$
\left|S_{1}^{\prime}\right| \leqslant \exp (G / H)
$$

and

$$
\sigma\left(S_{1}^{\prime}\right) \in \operatorname{ker}(\varphi)
$$


Let $S^{\prime \prime}=S_{1} \cdot S^{\prime} \cdot S_{1}^{\prime-1}$. By (3.8), (3.9), (3.10) and (3.11), we conclude that $\left|\operatorname{supp}\left(\varphi\left(S^{\prime \prime}\right)\right)\right| \geqslant$ $c+1$, a contradiction with (3.8). This proves the lemma.

From Lemma 17, we immediately obtain the following

Lemma 18. Let $r \in \mathbb{N}$, and let $G_{1}=C_{n_{1}}^{r}, G_{2}=C_{n_{2}}^{r}$ and $G=C_{n_{1} n_{2}}^{r}$. Suppose that the following conditions hold.

(i) $\frac{\eta\left(G_{1}\right)-1}{n_{1}-1}=\frac{\eta\left(G_{2}\right)-1}{n_{2}-1}=\frac{\eta(G)-1}{n_{1} n_{2}-1}=c$ for some $c \in \mathbb{N}$;

(ii) $G_{2}$ has Property $C$;

(iii) There exist $t_{1} \in\left[1, n_{2}-1\right], t_{2} \in\{1,2\}$ such that $t_{2} \leqslant t_{1}$ and such that $\left[\eta\left(G_{2}\right)-\right.$ $\left.t_{1}, \eta\left(G_{2}\right)-t_{2}\right] \subset C_{0}\left(G_{2}\right)$.

Then,

$$
\left[\eta(G)-t_{1}, \eta(G)-t_{2}\right] \subset C_{0}(G)
$$

\section{Some lower bounds on $\min \left\{C_{0}(G)\right\}$}

In this section we shall prove the following

Proposition 19. Let $G=C_{n}^{r}$ with $n \geqslant 3, r \geqslant 3$, and let $\alpha_{r} \equiv-2^{r-1}(\bmod n)$ with $\alpha_{r} \in[0, n-1]$. Then,

1. $C_{0}(G) \subset\left[\left(2^{r}-1\right)(n-1)-\alpha_{r}+1, \eta(G)-1\right]$ if $\alpha_{r} \neq 0$.

2. $C_{0}(G) \subset\left\{\left(2^{r}-1\right)(n-1)-n,\left(2^{r}-1\right)(n-1)-n+1\right\}$ if $\alpha_{r}=0$.

Note that $\alpha_{r} \neq 0$ if and only if $n \neq 2^{k}$, or $n=2^{k}$ and $r-1<k$; and $\alpha_{r}=0$ if and only if $n=2^{k}$ and $k \leqslant r-1$.

For every $r \in \mathbb{N}$, let

$$
G=C_{n}^{r}=<e_{1}>\oplus \cdots \oplus<e_{r}>
$$

with $\left\langle e_{i}>=C_{n}\right.$ for every $i \in[1, r]$, and let

$$
S_{r}=\prod_{\emptyset \neq I \subset[1, r]}\left(\sum_{i \in I} e_{i}\right)^{n-1} .
$$

We can regard $C_{n}^{r}$ as a subgroup of $C_{n}^{r+1}$ and therefore $S_{r+1}$ has the following decomposition

$$
S_{r+1}=S_{r}\left(S_{r}+e_{r+1}\right) e_{r+1}^{n-1} .
$$

Since the proof of Proposition 19 is somewhat long, we split the proof into lemmas begin with the following easy one

Lemma 20. $S_{r}$ is a short free sequence over $C_{n}^{r}$ of length $\left|S_{r}\right|=\left(2^{r}-1\right)(n-1)$ and of sum $\sigma\left(S_{r}\right)=-2^{r-1}\left(e_{1}+\cdots+e_{r}\right)=\alpha_{r}\left(e_{1}+\cdots+e_{r}\right)$.

Proof. Obviously. 
Lemma 21. Let $G=C_{n}^{r}$ with $r \geqslant 2$. Then for every $m \in[1, n-1]$ and every $i \in[1, r]$, the sequence $S_{r}\left(e_{i}^{m}\right)^{-1}\left(m_{i}\right)$ contains no short zero-sum subsequence.

Proof. Without loss of generality, we assume that $i=r$.

Assume to the contrary that $S_{r}\left(e_{r}^{m}\right)^{-1}\left(m e_{r}\right)$ contains a short zero-sum subsequence $U$. Since $S_{r}$ contains no short zero-sum subsequence we infer that $m e_{r} \mid U$. Therefore, $U=\left(m e_{r}\right) U_{0}\left(U_{1}+e_{r}\right) e_{r}^{k}$ with $U_{0} \mid S_{r-1}$ and $U_{1} \mid S_{r-1}$ and $k \in[0, n-1-m]$. It follows that $U_{0} U_{1}$ is zero-sum and $1 \leqslant\left|U_{0} U_{1}\right| \leqslant n-1$. Since every element in $\operatorname{supp}\left(S_{r-1}\right)$ occurs $n-1$ times in $S_{r-1}$, it follows from $\left|U_{0} U_{1}\right| \leqslant n-1$ that $U_{0} U_{1} \mid S_{r-1}$. Therefore, $U_{0} U_{1}$ is a short zero-sum subsequence of $S_{r-1}$, a contradiction with Lemma 20.

Let $A$ be a set of zero-sum sequences over $G$. Define

$$
\mathcal{L}(A)=\{|T|: T \in A\}
$$

In this section below we shall frequently use the following easy observation.

Lemma 22. Let $G$ be a finite abelian group, and let $a, b \in \mathbb{N}$ with $a \leqslant b$. If there exists a set $A$ of zero-sum short free sequences over $G$ such that $[a, b] \subset \mathcal{L}(A)$, then $C_{0}(G) \cap[a, b]=\emptyset$.

Proof. It immediately follows from the definition of $C_{0}(G)$.

Lemma 23. Let $G=C_{n}^{r}$ with $n, r \geqslant 3$. Then,

$$
\begin{aligned}
& \text { 1. } C_{0}(G) \cap\left[\left|S_{r}\right|-(3 n-3)-\alpha_{r},\left|S_{r}\right|-\alpha_{r}\right]=\emptyset \text { if } \alpha_{r} \neq 0 . \\
& \text { 2. } C_{0}(G) \cap\left[\left|S_{r}\right|-(3 n-3),\left|S_{r}\right|-(n+1)\right]=\emptyset \text { if } \alpha_{r}=0 .
\end{aligned}
$$

Proof. Recall that $\left|S_{r}\right|=\left(2^{r}-1\right)(n-1)$. We split the proof into three steps.

Step 1. In this step we shall prove that

$$
C_{0}(G) \cap\left[\left|S_{r}\right|-(3 n-3)-\alpha_{r},\left|S_{r}\right|-(n+1)-\alpha_{r}\right]=\emptyset
$$

no matter $\alpha_{r}=0$ or not.

Let

$$
A=\left\{S_{r}\left(\left(e_{1}+\cdots+e_{r}\right)^{\alpha_{r}} W e_{3}^{m}\right)^{-1}\left(m e_{3}\right): W \mid S_{2}, \sigma(W)=0, m \in[1, n-1]\right\} .
$$

It follows from Lemma 21 that every sequence in $A$ is zero-sum short free.

Since $\mathcal{L}\left(\left\{W: W \mid S_{2}, \sigma(W)=0\right\}\right)=[n+1,2 n-1]$, we conclude easily that

$$
\mathcal{L}(A)=\left[\left|S_{r}\right|-(3 n-3)-\alpha_{r},\left|S_{r}\right|-(n+1)-\alpha_{r}\right] .
$$

Now the result follows from Lemma 22 and Conclusion 2 follows.

Step 2. We show that $C_{0}(G) \cap\left[\left|S_{r}\right|-\left(n+\alpha_{r}\right),\left|S_{r}\right|-(r-1) \alpha_{r}\right]=\emptyset$ for $\alpha_{r} \neq 0$. 
Let

$$
A_{1}=\left\{S_{r}\left(\left(e_{1}+e_{2}\right)^{\alpha_{r}} e_{3}^{\alpha_{r}} \cdot \ldots \cdot e_{r}^{\alpha_{r}} e_{1}^{m}\right)^{-1}\left(m e_{1}\right): m \in[1, n-1]\right\}
$$

and

$$
A_{2}=\left\{S_{r}\left(\left(e_{1}+e_{2}\right)^{\alpha_{r}}\left(e_{1}+e_{3}\right) e_{3}^{\alpha_{r}-1} e_{4}^{\alpha_{r}} \cdot \ldots \cdot e_{r}^{\alpha_{r}} e_{1}^{n-1}\right)^{-1}\right\}
$$

It is easy to see that every sequence in $A_{1} \cup A_{2}$ is zero-sum short free by Lemma 21 and Lemma 20. Note that

$$
\begin{aligned}
\mathcal{L}\left(A_{1}\right) \cup \mathcal{L}\left(A_{2}\right) & =\left[\left|S_{r}\right|-(r-1) \alpha_{r}-n+2,\left|S_{r}\right|-(r-1) \alpha_{r}\right] \cup\left\{\left|S_{r}\right|-(r-1) \alpha_{r}-n+1\right\} \\
& =\left[\left|S_{r}\right|-(r-1) \alpha_{r}-n+1,\left|S_{r}\right|-(r-1) \alpha_{r}\right] .
\end{aligned}
$$

Since $r \geqslant 3$, we have $\left|S_{r}\right|-(r-1) \alpha_{r}-n+1 \leqslant\left|S_{r}\right|-\left(n+\alpha_{r}\right)$. Therefore, $\mathcal{L}\left(A_{1} \cup A_{2}\right)=$ $\mathcal{L}\left(A_{1}\right) \cup \mathcal{L}\left(A_{2}\right) \supset\left[\left|S_{r}\right|-\left(n+\alpha_{r}\right),\left|S_{r}\right|-(r-1) \alpha_{r}\right]$. Again the result follows from Lemma 22.

Step 3. We prove $C_{0}(G) \cap\left[\left|S_{r}\right|-(r-1) \alpha_{r},\left|S_{r}\right|-\alpha_{r}\right]=\emptyset$ for $\alpha_{r} \neq 0$.

Let

$$
\begin{gathered}
A=\left\{S_{r}\left(\left(e_{1}+\cdots+e_{r}\right)^{k_{1}}\left(e_{1} \cdot \ldots \cdot e_{r}\right)^{k_{2}}\left(e_{1}+\cdots+e_{k_{3}}\right) e_{k_{3}+1} \cdot \cdots \cdot e_{r}\right)^{-1}:\right. \\
\left.k_{1} \in\left[0, \alpha_{r}-1\right], k_{2} \in\left[0, \alpha_{r}-1\right], k_{1}+k_{2}=\alpha_{r}-1, k_{3} \in[1, r]\right\} .
\end{gathered}
$$

Then every sequence in $A$ is zero-sum short free by Lemma 21 and by Lemma 20, and

$$
\begin{aligned}
& =\left\{\left|S_{r}\right|-k_{1}-r k_{2}-1-\left(r-k_{3}\right): k_{1}+k_{2}=\alpha_{r}-1, k_{2} \in\left[0, \alpha_{r}-1\right], k_{3} \in[1, r]\right\} \\
& =\left\{\left|S_{r}\right|-\alpha_{r}-\left((r-1) k_{2}+\left(r-k_{3}\right)\right): k_{2} \in\left[0, \alpha_{r}-1\right], k_{3} \in[1, r]\right\} \\
& =\left[\left|S_{r}\right|-r \alpha_{r},\left|S_{r}\right|-\alpha_{r}\right] .
\end{aligned}
$$

Now the result follows from Lemma 22 and the proof is completed.

Lemma 24. Let $n, r \in \mathbb{N}$ with $n \geqslant 3$ and $r \geqslant 3$, and let $G=C_{n}^{r}$. If $\alpha_{r} \neq 0$ then $C_{0}(G) \subset\left[\left(2^{r}-1\right)(n-1)-\alpha_{r}+1, \eta(G)-1\right]$.

Proof. It suffices to show that $C_{0}(G) \cap\left[n+1,\left|S_{r}\right|-\alpha_{r}\right]=\emptyset$.

We proceed by induction on $r$. Suppose first that $r=3$.

By Lemma 23 and the definition of $C_{0}\left(C_{n}^{3}\right)$, we only need to prove

$$
C_{0}(G) \cap\left[D\left(C_{n}^{3}\right)+1,\left|S_{3}\right|-(3 n-3)-\alpha_{3}-1\right]=\emptyset .
$$

By Lemma 5 we have $D\left(C_{n}^{3}\right)+1 \geqslant 3 n-1$. So, it suffices to prove that

$$
C_{0}(G) \cap\left[3 n-1,\left|S_{3}\right|-(3 n-3)-\alpha_{3}-1\right]=C_{0}(G) \cap\left[3 n-1,4 n-4-\alpha_{3}-1\right]=\emptyset .
$$


If $n=3$, then $\left[3 n-1,4 n-4-\alpha_{3}-1\right]=\emptyset$ and the result follows.

Now assume $n \geqslant 4$. It follows from $\alpha_{3} \neq 0$ that $n \geqslant 5$. Thus, $\alpha_{3}=n-4$ and $\left[3 n-1,4 n-4-\alpha_{3}-1\right]=\{3 n-1\}$.

Let $T=\left(e_{1}+e_{2}\right)^{2}\left(e_{1}+e_{3}\right)^{n-1} e_{1}^{n-1} e_{2}^{n-2} e_{3}$. Then $T$ is zero-sum short free over $C_{n}^{3}$ of length $|T|=3 n-1$. Now the result follows from Lemma 22. This completes the proof for $r=3$.

Now assume that $r \geqslant 4$. By the induction hypothesis there exists a set $A_{r-1}$ of zero-sum short free sequences over $C_{n}^{r-1}$ such that

$$
\mathcal{L}\left(A_{r-1}\right)=\left[n+1,\left|S_{r-1}\right|-\alpha_{r-1}\right] .
$$

Recall that $C_{n}^{r-1} \subset C_{n}^{r}=C_{n}^{r-1} \oplus\left\langle e_{r}\right\rangle$. Let

$$
A_{r}=\left\{W_{2}\left(W_{1}+e_{r}\right) e_{r}^{\ell}: W_{1} \in A_{r-1}, W_{2} \in A_{r-1}, \ell \in[0, n-1],\left|W_{1}\right|+\ell \equiv 0(\bmod n)\right\} .
$$

Then, every sequence in $A_{r}$ is zero-sum short free over $C_{n}^{r}$ and

$$
\begin{aligned}
\mathcal{L}\left(A_{r}\right) & =\left\{\left|W_{2}\right|+\left|W_{1}\right|+\ell: W_{1} \in A_{r-1}, W_{2} \in A_{r-1}, \ell \in[0, n-1],\left|W_{1}\right|+\ell \equiv 0(\bmod n)\right\} \\
& =\left\{\left|W_{2}\right|+k n: W_{2} \in A_{r-1}, k \in\left[2,\left\lceil\frac{\left|S_{r-1}\right|-\alpha_{r-1}}{n}\right\rceil\right]\right. \\
& \supset\left[3 n+1,2\left|S_{r-1}\right|-2 \alpha_{r-1}\right] .
\end{aligned}
$$

It follows that

$$
\mathcal{L}\left(A_{r-1}\right) \cup \mathcal{L}\left(A_{r}\right) \supset\left[n+1,2\left|S_{r-1}\right|-2 \alpha_{r-1}\right] .
$$

Note that

$$
\begin{aligned}
2\left|S_{r-1}\right|-2 \alpha_{r-1} & =\left|S_{r}\right|-(n-1)-2 \alpha_{r-1} \\
& \geqslant\left|S_{r}\right|-3(n-1) .
\end{aligned}
$$

Therefore,

$$
\mathcal{L}\left(A_{r-1}\right) \cup \mathcal{L}\left(A_{r}\right) \supset\left[n+1,\left|S_{r}\right|-3(n-1)\right] .
$$

Now the result follows from Lemma 23.

Lemma 25. Let $n, r, k \in \mathbb{N}$ with $k \geqslant 2, r \geqslant k+1$ and $n=2^{k}$, and let $G=C_{n}^{r}$. Then, $C_{0}(G) \subset\left\{\left(2^{r}-1\right)(n-1)-n,\left(2^{r}-1\right)(n-1)-n+1\right\}$.

Proof. Since $r \geqslant k+1$ we have that $\alpha_{r}=0$.

By Lemma 7 we have

$$
\left|S_{r}\right|=\left(2^{r}-1\right)(n-1)=\eta(G)-1 .
$$

So, it suffices to show that $C_{0}(G) \cap([n+1, \eta(G)-(n+2)] \cup[\eta(G)-n+1, \eta(G)-1])=\emptyset$.

Since $r \geqslant k+1$ we have

$$
\sigma\left(S_{r}\right)=0 .
$$


Step 1. We show $C_{0}(G) \cap\left[n+1,\left|S_{r}\right|-(n+1)\right]=\emptyset$.

We proceed by induction on $r$. Suppose first that $r=k+1$.

If $r=k+1=3$, we only need to prove $C_{0}(G) \cap[3 n-1,4 n-5]=\emptyset$ by Lemma 23 and Lemma 5. Let

$$
\begin{aligned}
A= & \left\{\left(e_{1}+e_{2}+e_{3}\right)\left(e_{1}+e_{2}\right)^{n-1}\left(e_{1}+e_{3}\right)^{n-m}\left(e_{2}+e_{3}\right) e_{1}^{m} e_{2}^{n-1} e_{3}^{m-2}: m \in[2, n-1]\right\} \cup \\
& \left\{\left(e_{1}+e_{2}\right)^{2}\left(e_{1}+e_{3}\right)^{n-1} e_{1}^{n-1} e_{2}^{n-2} e_{3}\right\} .
\end{aligned}
$$

Then every sequence in $A$ is zero-sum short free and $\mathcal{L}(A)=[3 n-1,4 n-3]$ and we are done.

If $r=k+1>3$, we have $\alpha_{r-1} \neq 0$ and $r-1 \geqslant 3$, then by Lemma 24 there exists a set $A$ of zero-sum short free sequences over $C_{n}^{r-1}$ such that $\mathcal{L}(A) \supset\left[n+1,\left|S_{r-1}\right|-\alpha_{r-1}\right]$.

Let

$$
B=A \cup\left\{W_{2}\left(W_{1}+e_{r}\right) e_{r}^{\ell}: W_{1} \in A, W_{2} \in A, \ell \in[0, n-1],\left|W_{1}\right|+\ell \equiv 0(\bmod n)\right\} .
$$

Since

$$
\left|S_{r-1}\right|-\alpha_{r-1}+\left|S_{r-1}\right|-\alpha_{r-1}+\alpha_{r-1}-1=\left|S_{r}\right|-3 n / 2,
$$

we have $\mathcal{L}(B) \supset\left[n+1,\left|S_{r}\right|-3 n / 2\right]$. It follows from Lemma 23 that $C_{0}\left(C_{n}^{r}\right) \cap\left[n+1,\left|S_{r}\right|-\right.$ $(n+1)]=\emptyset$.

Now assume that $r>k+1$. By the induction hypothesis, we conclude that there exists a set $A$ of zero-sum short free sequences over $C_{n}^{r-1}$ such that $\mathcal{L}(A) \supset\left[n+1,\left|S_{r-1}\right|-(n+1)\right]$.

Define a set $B$ of zero-sum short free sequences over $C_{n}^{r}$ as follows

$$
B=\left\{W_{2}\left(W_{1}+e_{r}\right) e_{r}^{\ell}: W_{1} \in A, W_{2} \in A, \ell \in[0, n-1],\left|W_{1}\right|+\ell \equiv 0(\bmod n)\right\} .
$$

It is easy to see that

$$
\mathcal{L}(B) \supset\left[\left|S_{r-1}\right|-n, 2\left|S_{r-1}\right|-2(n+1)\right]=\left[\left|S_{r-1}\right|-n,\left|S_{r}\right|-(3 n+1)\right] .
$$

Let

$$
\begin{aligned}
& C_{1}=\left\{T: T \mid S_{2}, \sigma(T)=0\right\} \\
& C_{2}=\left\{\left(e_{1}+e_{3}\right)^{n-m} e_{1}^{m-1} e_{2}^{n-1}\left(e_{1}+e_{2}\right) e_{3}^{m}: m \in[1, n-1]\right\} ; \\
& C_{3}=\left\{\left(e_{1}+e_{2}\right)^{2}\left(e_{1}+e_{3}\right)^{n-1} e_{1}^{n-1} e_{2}^{n-2} e_{3}\right\} \\
& C_{4}=\left\{\left(e_{1}+e_{2}+e_{3}\right)\left(e_{1}+e_{2}\right)^{n-1}\left(e_{1}+e_{3}\right)^{n-m}\left(e_{2}+e_{3}\right) e_{1}^{m} e_{2}^{n-1} e_{3}^{m-2}: m \in[2, n-1]\right\} .
\end{aligned}
$$

Then every sequence in $\cup_{i=1}^{4} C_{i}$ is zero-sum short free. Clearly,

$$
\begin{aligned}
\mathcal{L}\left(C_{1}\right) & =[n+1,2 n-1] ; \\
\mathcal{L}\left(C_{2}\right) & =[2 n, 3 n-2] ; \\
\mathcal{L}\left(C_{3}\right) & =\{3 n-1\} ; \\
\mathcal{L}\left(C_{4}\right) & =[3 n, 4 n-3] .
\end{aligned}
$$


Let

$$
C=\cup_{i=1}^{4} C_{i}
$$

Then,

$$
\mathcal{L}(C) \supset[n+1,4 n-3] .
$$

Let

$$
D=\left\{S_{r} T^{-1}: T^{\prime} \in C\right\} .
$$

Then every sequence in $D$ is zero-sum short free, and

$$
\begin{aligned}
\mathcal{L}(D) & \supset\left[\left|S_{r}\right|-(4 n-3),\left|S_{r}\right|-(n+1)\right] \\
& \supset\left[\left|S_{r}\right|-3 n,\left|S_{r}\right|-(n+1)\right] .
\end{aligned}
$$

This completes the proof of Step 1.

Step 2. We prove $C_{0}(G) \cap[\eta(G)-n+1, \eta(G)-1]=\emptyset$.

Let

$$
A=\left\{S_{r}\left(e_{r}^{m}\right)^{-1}\left(m e_{r}\right): m \in[1, n-1]\right\} .
$$

Then every sequence in $A$ is zero-sum short free by Lemma 21, and

$$
\mathcal{L}(A)=\left[\left|S_{r}\right|-n+2,\left|S_{r}\right|\right]=[\eta(G)-n+1, \eta(G)-1] .
$$

This completes the proof.

Proof of Proposition 19. 1. It is just Lemma 24.

2. Since $\alpha_{r}=0$, we have $n=2^{k}$ for some $k \in[2, r-1]$, now the result follows from Lemma 25.

\section{$5 \quad$ On the groups $C_{3}^{r}$}

In this section we shall study $C_{0}(G)$ with focus on $G=C_{3}^{r}$.

Proposition 26. Let $r, t \in \mathbb{N}$. Then,

1. $C_{0}\left(C_{3}^{3}\right) \subset\left[\eta\left(C_{3}^{3}\right)-4, \eta\left(C_{3}^{3}\right)-1\right]$.

2. $C_{0}\left(C_{5}^{3}\right) \subset\left[\eta\left(C_{5}^{3}\right)-5, \eta\left(C_{5}^{3}\right)-1\right]$.

3. $C_{0}\left(C_{2^{t}}^{r}\right) \subset \begin{cases}{\left[\eta\left(C_{2^{t}}^{r}\right)-\left(2^{t}-2^{r-1}\right), \eta\left(C_{2^{t}}^{r}\right)-1\right],} & \text { if } r \leqslant t, \\ {\left[\eta\left(C_{2^{t}}^{r}\right)-\left(2^{t}+1\right), \eta\left(C_{2^{t}}^{r}\right)-2^{t}\right],} & \text { if } \quad r>t .\end{cases}$

4. $C_{0}\left(C_{6}^{3}\right) \subset\left\{\eta\left(C_{6}^{3}\right)-2, \eta\left(C_{6}^{3}\right)-1\right\}$.

Proof. Conclusions 1, 2 and 4 follow from Lemma 7 and Proposition 19. So, it remains to prove Conclusion 3. If $r \leqslant t$ then applying Proposition 19 with $\alpha_{r}=2^{t}-2^{r-1}$, it follows from Conclusion 6 of Lemma 7 that $C_{0}\left(C_{2^{t}}^{r}\right) \subset\left[\left(2^{r}-1\right)\left(2^{t}-1\right)-\left(2^{t}-2^{r-1}\right)+1, \eta\left(C_{2^{t}}^{r}\right)-1\right]=$ $\left[\eta\left(C_{2^{t}}^{r}\right)-\left(2^{t}-2^{r-1}\right), \eta\left(C_{2^{t}}^{r}\right)-1\right]$. If $r>t$ then applying Proposition 19 with $\alpha_{r}=0$ we get, $C_{0}\left(C_{2^{t}}^{r}\right) \subset\left[\eta\left(C_{2^{t}}^{r}\right)-\left(2^{t}+1\right), \eta\left(C_{2^{t}}^{r}\right)-2^{t}\right]$. 
Lemma 27. Let $G=C_{3}^{r}$ with $r \geqslant 3$, and let $S$ be a sequence over $G$. Then,

1. If $S$ is a short free sequence over $G$ of length $|S|=\eta(G)-1$, then $\sum_{\leqslant 2}(S)=C_{3}^{r} \backslash\{0\}$.

2. Let $T$ be a square free and short free sequence over $G$, and let $S=T^{2}$. Then, for every $g \in \operatorname{supp}(S)$ we have, $\sum_{\leqslant 2}\left(S \cdot g^{-1}\right)=\sum_{\leqslant 2}(S) \backslash\{2 g\}$.

3. If every short free sequence of length $\eta(G)-1$ has sum zero, then $\eta(G)-2 \in C_{0}(G)$.

Proof. Conclusions 1 and 2 are obvious.

To prove Conclusion 3, we assume to the contrary that $\eta(G)-2 \notin C_{0}(G)$, i.e., there exists a zero-sum short free sequence $S$ over $G$ of length $|S|=\eta(G)-2$. By Lemma 7 , we have $\eta(G)-2=2(f(G)-2)+1$. This forces that $S=g_{1}^{2} \cdot \ldots \cdot g_{f(G)-2}^{2} \cdot g_{f(G)-1}$ for some distinct elements $g_{1}, \ldots, g_{f(G)-1}$ with $g_{1} \cdot \ldots \cdot g_{f(G)-1}$ contains no short zero-sum subsequence. Put $T=S \cdot g_{f(G)-1}$. Then $|T|=\eta(G)-1$. But $T$ contains no short zero-sum subsequence and $\sigma(T)=g_{f(G)-1} \neq 0$, a contradiction.

Lemma 28. Every short free sequence over $C_{3}^{3}$ of length 16 has sum zero.

Proof. Let $S$ be an arbitrary short free sequence over $C_{3}^{3}$ of length $|S|=16$. From Lemma 7 we obtain that $S=T^{2}$, where $T$ is a square free and short free sequence over $C_{3}^{3}$ of length 8. It follows from Lemma 9 that $\sigma(T)=0$. Therefore, $\sigma(S)=2 \sigma(T)=0$.

Lemma 29. The following two conclusions hold.

1. $\{14,15\}=\left\{\eta\left(C_{3}^{3}\right)-3, \eta\left(C_{3}^{3}\right)-2\right\} \subset C_{0}\left(C_{3}^{3}\right)$.

2. $\{37,38\}=\left\{\eta\left(C_{3}^{4}\right)-2, \eta\left(C_{3}^{4}\right)-1\right\} \subset C_{0}\left(C_{3}^{4}\right)$.

Proof. 1. The conclusion $14 \in C_{0}\left(C_{3}^{3}\right)$ is due to Emde Boas and D. Kruyswijk [7]. Now $15 \in C_{0}\left(C_{3}^{3}\right)$ follows from Conclusion 3 of Lemma 7, Lemma 27 and Lemma 28.

2. Denote by $U$ the square free sequence over $C_{3}^{4}$ given in Lemma 10. It follows from Conclusion 4 of Lemma 7 that $U$ is a square free sequence of maximum length which contains no zero-sum subsequence of length 3.

Choose an arbitrary square free sequence $T$ over $C_{3}^{4}$ of length $f\left(C_{3}^{4}\right)-1$ such that $T$ contains no short zero-sum subsequence. By Lemma 7, we have $|T|=19$.

Claim. $\sigma(T) \notin-\operatorname{supp}(T) \cup\{0\}$.

Proof of the claim. Put $S=T \cdot 0$. It follows from Conclusion 4 of Lemma 7 that $S$ is a square free sequence over $C_{3}^{4}$ of maximum length which contains no zero-sum subsequence of length 3. By Lemma 8, there exists an automorphism $\varphi$ of $C_{3}^{4}$ and some $g \in C_{3}^{4}$ such that $S=\varphi(U-g)$. Since $0 \mid S$, it follows that $g \mid U$. Thus, $\sigma(T)=\sigma(S)=\sigma(\varphi(U-g))=$ $\varphi(\sigma(U-g))=\varphi(\sigma(U)-20 g)=\varphi(\sigma(U)+g)$. It is easy to check that $\sigma(U)=\left(\begin{array}{l}2 \\ 2 \\ 2 \\ 2\end{array}\right)$. Since $-\sigma(U)=\left(\begin{array}{l}1 \\ 1 \\ 1\end{array}\right) \notin \operatorname{supp}(U)$, it follows that $-\sigma(T)=-\varphi(\sigma(U)+g)=\varphi(-\sigma(U)-g) \notin$ $\varphi(\operatorname{supp}(U)-g)=\operatorname{supp}(S)=\operatorname{supp}(T) \cup\{0\}$. This proves the claim. 
From Conclusions 4, 9, 10 of Lemma 7 and the above claim, we derive that every short free sequence over $C_{3}^{4}$ of length $\eta\left(C_{3}^{4}\right)-1=38$ has a nonzero sum. This is equivalent to that every zero-sum sequence over $C_{3}^{4}$ of length $\eta\left(C_{3}^{4}\right)-1$ contains a short zero-sum subsequence. Hence, $38=\eta\left(C_{3}^{4}\right)-1 \in C_{0}\left(C_{3}^{4}\right)$.

Suppose that $37=\eta\left(C_{3}^{4}\right)-2 \notin C_{0}(G)$, that is, there exists a zero-sum short free sequence $V$ over $C_{3}^{4}$ of length $|V|=\eta\left(C_{3}^{4}\right)-2=37$. Since $\mathrm{v}_{g}(V) \leqslant 2$ for every $g \in \operatorname{supp}(V)$, we have $|\operatorname{supp}(V)| \geqslant 19$. On the other hand, by Conclusion 4 and 9 of Lemma 7 , we can derive that $|\operatorname{supp}(V)| \leqslant f\left(C_{3}^{4}\right)-1=\frac{\eta\left(C_{3}^{4}\right)-1}{2}=19$. Thus, $V=W^{2} h^{-1}$, where $h \mid W$ and $W$ is a square free and short free sequence over $G$ of length $f\left(C_{3}^{4}\right)-1=19$. It follows from $\sigma(V)=0$ that $\sigma(W)=-h \in-\operatorname{supp}(W)$, a contradiction with the claim above.

Proposition 30. Let $G=C_{3}^{r}$ with $r \geqslant 3$. If there is a short free sequence $S$ over $G$ of length $|S|=\eta(G)-1$ such that $\sigma(S) \neq 0$, then

$$
\begin{aligned}
& \text { 1. }\left|\{\eta(G)-2, \eta(G)-3\} \cap C_{0}(G)\right| \leqslant 1 . \\
& \text { 2. }\left|\{\eta(G)-3, \eta(G)-4\} \cap C_{0}(G)\right| \leqslant 1 .
\end{aligned}
$$

Proof. 1. Since $\sigma(S) \neq 0$, it follows from Lemma 27 that there exists a subsequence $W$ of $S$ of length $|W| \in\{1,2\}$ such that $\sigma(S)=\sigma(W)$. Therefore, $\sigma\left(S \cdot W^{-1}\right)=0$, $\left|S \cdot W^{-1}\right| \in\{\eta(G)-3, \eta(G)-2\}$ and $S \cdot W^{-1}$ contains no short zero-sum subsequence. Hence, $\eta(G)-2 \notin C_{0}(G)$ or $\eta(G)-3 \notin C_{0}(G)$.

2. By Conclusion 10 of Lemma 7 , we have that $S=T^{2}$, where $T$ is a square free sequence over $G$. Choose $g \in \operatorname{supp}(S)$ such that $\sigma\left(S \cdot g^{-1}\right) \neq 0$. Since $\sigma\left(S \cdot g^{-1}\right)=\sigma(S)-g \neq 2 g$, it follows from Conclusion 2 of Lemma 27 that $\sigma\left(S \cdot g^{-1}\right) \in \sum_{\leqslant 2}\left(S \cdot g^{-1}\right)=C_{3}^{r} \backslash\{0,2 g\}$. Similarly to Conclusion 1 , we infer that $\eta(G)-3 \notin C_{0}(G)$ or $\eta(G)-4 \notin C_{0}(G)$.

Proposition 31. $C_{0}\left(C_{3}^{4}\right)=\{37,38\}$.

Proof. By Proposition 19, we have

$$
C_{0}\left(C_{3}^{4}\right) \subset\left[30, \eta\left(C_{3}^{4}\right)-1\right]=[30,38] .
$$

We show next that

$$
[30,36] \cap C_{0}\left(C_{3}^{4}\right)=\emptyset
$$

Put

$$
\begin{aligned}
& T_{2}=\left(\begin{array}{l}
2 \\
2 \\
2 \\
2
\end{array}\right)^{2} ; \\
& T_{3}=\left(\begin{array}{l}
2 \\
2 \\
0 \\
0
\end{array}\right)\left(\begin{array}{l}
0 \\
0 \\
2 \\
2
\end{array}\right)\left(\begin{array}{l}
2 \\
2 \\
2 \\
2
\end{array}\right) ;
\end{aligned}
$$




$$
\begin{aligned}
T_{4} & =\left(\begin{array}{l}
2 \\
0 \\
0 \\
0
\end{array}\right)\left(\begin{array}{l}
0 \\
2 \\
0 \\
0
\end{array}\right)\left(\begin{array}{l}
0 \\
0 \\
2 \\
2
\end{array}\right)\left(\begin{array}{l}
2 \\
2 \\
2 \\
2
\end{array}\right) ; \\
T_{5} & =\left(\begin{array}{l}
2 \\
0 \\
0 \\
0
\end{array}\right)\left(\begin{array}{l}
0 \\
2 \\
0 \\
0
\end{array}\right)\left(\begin{array}{l}
0 \\
0 \\
2 \\
2
\end{array}\right)\left(\begin{array}{l}
0 \\
2 \\
2 \\
2
\end{array}\right) ; \\
T_{6} & =\left(\begin{array}{l}
0 \\
2 \\
0 \\
0
\end{array}\right)\left(\begin{array}{l}
2 \\
2 \\
2 \\
2
\end{array}\right)\left(\begin{array}{l}
0 \\
1 \\
0 \\
2
\end{array}\right)\left(\begin{array}{l}
1 \\
2 \\
0 \\
2
\end{array}\right)\left(\begin{array}{l}
2 \\
1 \\
0 \\
2
\end{array}\right) ; \\
T_{7} & =\left(\begin{array}{l}
2 \\
0 \\
0 \\
0
\end{array}\right)\left(\begin{array}{l}
0 \\
2 \\
0 \\
0
\end{array}\right)\left(\begin{array}{l}
1 \\
0 \\
2 \\
0
\end{array}\right)\left(\begin{array}{l}
0 \\
1 \\
2 \\
0
\end{array}\right)\left(\begin{array}{l}
1 \\
2 \\
2 \\
0
\end{array}\right)\left(\begin{array}{l}
0 \\
0 \\
2 \\
2
\end{array}\right)\left(\begin{array}{l}
0 \\
2 \\
2 \\
2
\end{array}\right) ; \\
T_{8}= & \left(\begin{array}{l}
2 \\
0 \\
0 \\
0
\end{array}\right)\left(\begin{array}{l}
0 \\
2 \\
0 \\
0
\end{array}\right)\left(\begin{array}{l}
1 \\
0 \\
2 \\
0
\end{array}\right)\left(\begin{array}{l}
0 \\
1 \\
2 \\
0
\end{array}\right)\left(\begin{array}{l}
1 \\
2 \\
2 \\
0 \\
0
\end{array}\right)\left(\begin{array}{l}
2 \\
2 \\
2
\end{array}\right) \cdot
\end{aligned}
$$

Let $U$ be the square free sequence given in Lemma 10. Then $\sigma(U)=\left(\begin{array}{l}2 \\ 2 \\ 2 \\ 2\end{array}\right)$. Let $S=$ $U^{2} \cdot 0^{-2}$. We see that $S$ is a short free sequence of length $38=\eta\left(C_{3}^{4}\right)-1$. By removing $T_{i}$ from $S$, we obtain that the resulting sequence $S_{i}$ is a zero-sum short free sequence of length $\eta(G)-i-1=38-i$. This proves (5.2). Combining (5.1), (5.2) and Lemma 29, we conclude that $C_{0}\left(C_{3}^{4}\right)=\{\eta(G)-2, \eta(G)-1\}=\{37,38\}$.

\section{Proof of Theorem 2}

In this section we shall prove Theorem 2 and we need the following lemma.

Lemma 32. Let $p$ be a prime and let $H$ be a finite abelian $p$-group such that $p^{n} \geqslant D(H)$. Then,

1. Every sequence $S$ over $C_{p^{n}} \oplus H$ of length $|S|=2 p^{n}+D(H)-2$ contains a zero-sum subsequence $T$ of length $|T| \in\left\{p^{n}, 2 p^{n}\right\}$.

2. $\eta\left(C_{m p^{n}} \oplus H\right) \leqslant m p^{n}+p^{n}+D(H)-2$. 
Proof. 1. Let $S=g_{1} \cdot \ldots \cdot g_{\ell}$ be a sequence over $G=C_{p^{n}} \oplus H$ of length $\ell=|S|=$ $2 p^{n}+D(H)-2$. Let $\alpha_{i}=\left(\begin{array}{c}1 \\ g_{i}\end{array}\right) \in C_{p^{n}} \oplus C_{p^{n}} \oplus H$ with $1 \in C_{p^{n}}$. By Conclusion 10 of Lemma $13, \alpha_{1} \cdot \ldots \cdot \alpha_{\ell}$ is a sequence over $C_{p^{n}} \oplus G$ of length $\ell=p^{n}+p^{n}+D(H)-2=D\left(C_{p^{n}} \oplus G\right)$. Therefore, $\alpha_{1} \cdot \ldots \cdot \alpha_{\ell}$ contains a nonempty zero-sum subsequence $W$ (say). By the making of $\alpha_{i}$ we infer that $|W|=p^{n}$ or $|W|=2 p^{n}$. Let $T$ be the subsequence of $S$ which corresponds to $W$. Then $T$ is a zero-sum subsequence of $S$ of length $|T| \in\left\{p^{n}, 2 p^{n}\right\}$.

2. We first consider the case that $m=1$. Let $G=C_{p^{n}} \oplus H$. We want to prove that $\eta(G) \leqslant 2 p^{n}+D(H)-2$.

Let $S=g_{1} \cdot \ldots \cdot g_{\ell}$ be a sequence over $G=C_{p^{n}} \oplus H$ of length $\ell=|S|=2 p^{n}+D(H)-2$. We need to show that $S$ contains a short zero-sum subsequence. It follows from Conclusion 1 that $S$ contains a zero-sum subsequence $T$ of length $|T| \in\left\{p^{n}, 2 p^{n}\right\}$. If $|T|=p^{n}$ then $T$ itself is a short zero-sum sequence over $G$ and we are done. Otherwise, since $p^{n} \geqslant D(H)$, it follows from Conclusion 3 of Lemma 13 that $|T|=2 p^{n}>p^{n}+D(H)-1=D(G)$. Therefore, $T$ contains a nonempty proper zero-sum subsequence $T^{\prime}$. Now either $T^{\prime}$ or $T T^{\prime-1}$ is a short zero-sum subsequence of $S$. This proves that $\eta\left(C_{p^{n}} \oplus H\right) \leqslant 2 p^{n}+D(H)-2$. By Lemma 12, we have

$$
\begin{aligned}
\eta\left(C_{m p^{n}} \oplus H\right) & \leqslant\left(\eta\left(C_{m}\right)-1\right) \exp \left(C_{p^{n}} \oplus H\right)+\eta\left(C_{p^{n}} \oplus H\right) \\
& \leqslant(m-1) p^{n}+2 p^{n}+D(H)-2 \\
& =m p^{n}+p^{n}+D(H)-2 .
\end{aligned}
$$

Lemma 33. Let $G$ be a finite abelian group. Then $[D(G)+1, \min \{2 \exp (G)+1, \eta(G)-$ $1\}] \subset C_{0}(G)$.

Proof. If $[D(G)+1, \min \{2 \exp (G)+1, \eta(G)-1\}]=\emptyset$ then the conclusion of this lemma hold true trivially. Now assume that $[D(G)+1, \min \{2 \exp (G)+1, \eta(G)-1\}] \neq \emptyset$. Let $S$ be an arbitrary zero-sum sequence over $G$ of length $|S| \in[D(G)+1, \min \{2 \exp (G)+1, \eta(G)-1\}]$. It suffices to show that $S$ contains a short zero-sum subsequence. Since $|S| \geqslant D(G)+1$, it follows that $S$ contains a zero-sum subsequence $T$ of length $|T| \in[1,|S|-1]$. Then $\sigma\left(S T^{-1}\right)=0$. Since $|S| \leqslant 2 \exp (G)+1$, we infer that $|T| \in[1, \exp (G)]$ or $\left|S T^{-1}\right| \in$ $[1, \exp (G)]$. This proves the lemma.

Proof of Theorem 2, 1. By the definition of $C_{0}(G)$ we have, $C_{0}(G) \subset[D(G)+1, \eta(G)-1]$. So, we need to show

$$
[D(G)+1, \eta(G)-1] \subset C_{0}(G) .
$$

Suppose first that

$$
G=C_{n} \oplus C_{n} .
$$

By Conclusions 1 and 4 of Lemma 13, we have $D(G)=2 n-1$ and $\eta(G)=3 n-2$. Let $S$ be a zero-sum sequence over $G$ of length $|S| \in[2 n, 3 n-3]$. We need to show $S$ contains a short zero-sum subsequence. We may assume that

$$
\mathrm{v}_{0}(S)=0 .
$$


Let $T=S \cdot 0^{3 n-2-|S|}$. Then $|T|=3 n-2$ and $T$ contains a zero-sum subsequence $T^{\prime}$ of length $\left|T^{\prime}\right| \in\{n, 2 n\}$ by Lemma 11. If $\left|T^{\prime}\right|=n$ then $T^{\prime} 0^{-v_{0}\left(T^{\prime}\right)}$ is a short zero-sum subsequence of $S$ and we are done. So, we may assume that $\left|T^{\prime}\right|=2 n$. Let $T^{\prime \prime}=T T^{\prime-1}$. Now $T^{\prime \prime}$ is a zero-sum subsequence of $T$ of length $\left|T^{\prime \prime}\right|=n-2$. If $T^{\prime \prime}$ contains at least one nonzero element then $T^{\prime \prime} 0^{-v_{0}\left(T^{\prime \prime}\right)}$ is a short zero-sum subsequence of $S$ and we are done. So, we may assume that $T^{\prime \prime}=0^{n-2}$. This forces that $T^{\prime}=S$. It follows from $D(G)=2 n-1$ that $S$ contains a zero-sum subsequence $S_{0}$ of length $\left|S_{0}\right| \in[1,2 n-1]$. Therefore, either $S_{0}$ or $S S_{0}^{-1}$ is a short zero-sum subsequence of $S$.

Now suppose that

$$
G=C_{n} \oplus C_{m}
$$

with $n \mid m$ and

$$
n<m \text {. }
$$

By Conclusions 1 and 4 of Lemma 13, we have that $D(G)=n+m-1<2 m$ and $2 m+1>2 n+m-2=\eta(G)$. It follows from Lemma 33 that $[D(G)+1, \eta(G)-1] \subset C_{0}(G)$.

2. By Conclusion 2 of Lemma 13 and Conclusion 2 of Lemma 32, we have that $D\left(C_{m p^{n}} \oplus\right.$ $H)=m p^{n}+D(H)-1$ and $\eta\left(C_{m p^{n}} \oplus H\right) \leqslant m p^{n}+p^{n}+D(H)-2$.

Suppose $m \geqslant 2$. Then $\eta\left(C_{m p^{n}} \oplus H\right) \leqslant 2 m p^{n}$. Similarly to the proof of Conclusion 1 , we can prove that $\left[D\left(C_{m p^{n}} \oplus H\right)+1, \eta\left(C_{m p^{n}} \oplus H\right)-1\right] \subset C_{0}(G)$, and we are done. So, we may assume

$$
m=1 .
$$

Then $\eta\left(C_{p^{n}} \oplus H\right) \leqslant 2 p^{n}+D(H)-2$ and the proof is similar to that of 1 by using Conclusion 1 of Lemma 32 .

3. It is just Proposition 31.

4. Observe that $\sum_{g \in C_{2}^{r} \backslash\{0\}} g=0$. Then, any square free sequence $S$ over $C_{2}^{r}$ with $\mathrm{v}_{0}(S)=0$ and $|S| \in\left\{2^{r}-3,2^{r}-2\right\}$ must have a nonzero sum. It follows from Conclusion 6 of Lemma 7 that $\left\{\eta\left(C_{2}^{r}\right)-3, \eta\left(C_{2}^{r}\right)-2\right\}=\left\{2^{r}-3,2^{r}-2\right\} \subset C_{0}\left(C_{2}^{r}\right)$. So, $C_{0}\left(C_{2}^{r}\right)=\left\{2^{r}-3,2^{r}-2\right\}=$ $\left\{\eta\left(C_{2}^{r}\right)-3, \eta\left(C_{2}^{r}\right)-2\right\}$ follows from Proposition 19.

\section{Proof of Theorem 3}

Lemma 34. If $\frac{\eta\left(C_{m}^{r}\right)-1}{m-1}=\frac{\eta\left(C_{n}^{r}\right)-1}{n-1}=c$ for some $c \in \mathbb{N}$ and if $\eta\left(C_{m n}^{r}\right) \geqslant c(m n-1)+1$ then $\eta\left(C_{m n}^{r}\right)=c(m n-1)+1$.

Proof. The lemma follows from Lemma 12.

Lemma 35. $C_{2^{t}}^{r}$ has Property $C$.

Proof. It follows from Lemma 4 and Conclusion 6 of Lemma 7 by induction on $t$.

Proposition 36. Let $n=3 m$, where $m$ is an odd positive integer. Then,

1. If $\eta\left(C_{m}^{3}\right)=8 m-7$ then $\eta\left(C_{n}^{3}\right)-2 \in C_{0}\left(C_{n}^{3}\right)$. 
2. If $\eta\left(C_{m}^{4}\right)=19 m-18$ then $\left\{\eta\left(C_{n}^{4}\right)-2, \eta\left(C_{n}^{4}\right)-1\right\} \subset C_{0}\left(C_{n}^{4}\right)$.

Proof. 1. By Conclusion 3 of Lemma 7 and Lemma 12, we have

$$
\begin{aligned}
\eta\left(C_{n}^{3}\right) & \leqslant\left(\eta\left(C_{3}^{3}\right)-1\right) \cdot m+\eta\left(C_{m}^{3}\right) \\
& =16 m+8 m-7 \\
& =8 n-7 .
\end{aligned}
$$

Combined with Conclusion 1 of Lemma 7, we have

$$
\frac{\eta\left(C_{n}^{3}\right)-1}{n-1}=\frac{\eta\left(C_{m}^{3}\right)-1}{m-1}=\frac{\eta\left(C_{3}^{3}\right)-1}{3-1}=8 .
$$

Now we show $\eta\left(C_{n}^{3}\right)-2 \in C_{0}\left(C_{n}^{3}\right)$ by applying Lemma 18 with $G_{2}=C_{3}^{3}$ and $t_{1}=t_{2}=2$. Conditions (i)-(iii) of Lemma 18 are verified by (7.1), Conclusion 10 of Lemma 7, and Conclusion 1 of Lemma 29 respectively. We are done.

2. The proof is similar to that of Conclusion 1.

Proposition 37. Let $\alpha, \beta \in \mathbb{N}_{0}$ with $\alpha \geqslant 1$. Then,

1. If $\alpha+\beta \geqslant 2$ then $\left\{\eta\left(C_{3^{\alpha} 5^{\beta}}^{3}\right)-2, \eta\left(C_{3^{\alpha} 5^{\beta}}^{3}\right)-1\right\} \subset C_{0}\left(C_{3^{\alpha} 5^{\beta}}^{3}\right)$.

2. $\left\{\eta\left(C_{3^{\alpha}}^{4}\right)-2, \eta\left(C_{3^{\alpha}}^{4}\right)-1\right\} \subset C_{0}\left(C_{3^{\alpha}}^{4}\right)$.

Proof. 1. By Conclusions 1, 3 and 5 of Lemma 7 and Lemma 34, we conclude that

$$
\frac{\eta\left(C_{3^{s} 5^{t}}^{3}\right)-1}{3^{s} 5^{t}-1}=8
$$

for every $s, t \in \mathbb{N}_{0}$ with $s+t \geqslant 1$. Combined with Proposition 36 , we have $\eta\left(C_{3^{\alpha} 5^{\beta}}^{3}\right)-2 \in$ $C_{0}\left(C_{3^{\alpha} 5^{\beta}}^{3}\right)$.

By Lemma 4, Conclusions 8, 10 of Lemma 7 and (7.2), we have $C_{3^{\alpha} 5^{\beta}}^{3}$ has Property C. Since $\alpha+\beta \geqslant 2$, we have $8<3^{\alpha} 5^{\beta}$. Therefore, it follows from (7.2) and Lemma 16 that $\eta\left(C_{3^{\alpha} 5^{\beta}}^{3}\right)-1 \in C_{0}\left(C_{3^{\alpha} 5^{\beta}}^{3}\right)$. We are done.

2. By Conclusion 2 of Lemma 29, we need only to consider the case that $\alpha>1$. By Conclusions 2 and 4 of Lemma 7 and Lemma 34, we can derive

$$
\frac{\eta\left(C_{3^{\alpha-1}}^{4}\right)-1}{3^{\alpha-1}-1}=19
$$

Combined with Conclusion 2 of Proposition 36, we have $\left\{\eta\left(C_{3^{\alpha}}^{4}\right)-2, \eta\left(C_{3^{\alpha}}^{4}\right)-1\right\} \subset$ $C_{0}\left(C_{3^{\alpha}}^{4}\right)$, done.

Proposition 38. Let $m=3^{\alpha} 5^{\beta}$ with $\alpha \in \mathbb{N}$ and $\beta \in \mathbb{N}_{0}$. Let $n \geqslant 65$ be an odd positive integer such that $C_{p}^{3}$ has Property $D_{0}$ with respect to 9 for all prime divisors $p$ of $n$. If

$$
m \geqslant \frac{6 \times 5^{7} n^{17}}{\left(n^{2}-7\right) n-64}+3
$$

then $\eta\left(C_{m n}^{3}\right)-2 \in C_{0}\left(C_{m n}^{3}\right)$. 
Proof. Let $m^{\prime}=\frac{m}{3}$. Then $m^{\prime}=3^{\alpha-1} 5^{\beta} \geqslant \frac{2 \times 5^{7} n^{17}}{\left(n^{2}-7\right) n-64}$ and $\alpha-1 \geqslant 0$.

By Lemma 15 and Lemma 14 we have $s\left(C_{m^{\prime} n}^{3}\right)=9 m^{\prime} n-8$ and $\eta\left(C_{m^{\prime} n}^{3}\right) \leqslant 8 m^{\prime} n-7$. It follows from Lemma 7 that $\eta\left(C_{m^{\prime} n}^{3}\right)=8 m^{\prime} n-7$. Since $\eta\left(C_{3}^{3}\right)=8 \times 3-7$ and $\eta\left(C_{m^{\prime} n}^{3}\right)=8 m^{\prime} n-7$, it follows from Lemma 34 that $\eta\left(C_{m n}^{3}\right)=8 m n-7$. What's more, $C_{3}^{3}$ has Property $C$ and $\eta\left(C_{3}^{3}\right)-2 \in C_{0}\left(C_{3}^{3}\right)$ by Lemma 29. Therefore, $\eta\left(C_{m n}^{3}\right)-2 \in C_{0}\left(C_{m n}^{3}\right)$ by Lemma 18 .

\section{Proof of Theorem 3.}

1. If $a \geqslant 1$ then it follows from Proposition 37 and Lemma 29. Now assume $b \geqslant 2$. Since $\eta\left(C_{3^{a} 5^{b}}^{3}\right)=8\left(3^{a} 5^{b}-1\right)+1$, it follows from Lemma 16 that $\eta\left(C_{3^{a} 5^{b}}^{3}\right)-1 \in C_{0}\left(C_{3^{a} 5^{b}}^{3}\right)$.

2. Let $G_{1}=C_{3 \times 2^{a-3}}^{3}$ and $G_{2}=C_{8}^{3}$. By Lemma 35, Conclusions 6, 7 and 8 of Lemma 7 , we have that $\eta\left(G_{1}\right)=7\left(3 \times 2^{a-3}-1\right)+1, \eta\left(G_{2}\right)=7 \times(8-1)+1$ and $G_{2}$ has Property C. Therefore, $\eta\left(C_{8}^{3}\right)-1 \in C_{0}\left(C_{8}^{3}\right)$ by Lemma 16 . So, $\eta\left(C_{3 \times 2^{a}}^{3}\right)-1 \in C_{0}\left(C_{3 \times 2^{a}}^{3}\right)$ by Lemma 18.

3. The result follows from Proposition 37.

4. Let $G=C_{2^{a}}^{r}$ with $3 \leqslant r \leqslant a$. By Lemma 35 and Conclusions 6 of Lemma 7 , we have $\eta\left(C_{2^{a}}^{r}\right)=\left(2^{r}-1\right)\left(2^{a}-1\right)+1$ and $C_{2^{a}}^{r}$ has Property C. Since $2^{r}-1<2^{a}$, it follows from Lemma 16 that $\eta\left(C_{2^{a}}^{r}\right)-1 \in C_{0}\left(C_{2^{a}}^{r}\right)$.

If $G=C_{2}^{r}$ and $r \geqslant 3$, then it follows from Conclusion 4 of Theorem 2.

5. Let $m=3^{n_{1}} 5^{n_{2}}$ and $n=7^{n_{3}} 11^{n_{4}} 13^{n_{5}}$. It follows from $n_{3}+n_{4}+n_{5} \geqslant 3$ that $n>65$. By the hypothesis of $n_{1}+n_{2} \geqslant 11+34\left(n_{3}+n_{4}+n_{5}\right)$ we infer that, $m=3^{n_{1}} 5^{n_{2}} \geqslant 3^{n_{1}+n_{2}} \geqslant$ $3^{11} 3^{34\left(n_{3}+n_{4}+n_{5}\right)}>4 \times 5^{8} \times 13^{14\left(n_{3}+n_{4}+n_{5}\right)} \geqslant 4 \times 5^{8} n^{14}>\frac{6 \times 5^{7} n^{17}}{\left(n^{2}-7\right) n-64}+3$. Since it has been proved that every prime $p \in\{3,5,7,11,13\}$ has Property $D_{0}$ with respect to 9 in [8], it follows from Proposition 38 that $\eta\left(C_{k}^{3}\right)-2 \in C_{0}\left(C_{k}^{3}\right)$.

\section{Concluding Remarks and Open Problems}

Proposition 39. Let $G$ be a non-cyclic finite abelian group with $\exp (G)=n$. Then $C_{0}(G) \cup\{\eta(G)\}$ doesn't contain $n+1$ consecutive integers.

Proof. Assume to contrary that $[t, t+n] \subset C_{0}(G) \cup\{\eta(G)\}$ for some $t \in \mathbb{N}$. By the definition of $C_{0}(G)$ we have that $t+n-1<\eta(G)$. So, we can choose a short free sequence $T$ over $G$ of length $|T|=t+n-1$. It follows from $t+n-1 \in C_{0}(G) \cup\{\eta(G)\}$ that $\sigma(T) \neq 0$. Let $g=\sigma(T)$ and let $S=T \cdot(-g)$. Since $|S|=t+n \in C_{0}(G) \cup\{\eta(G)\}, S$ contains a short zero-sum subsequence $U$ with $(-g) \mid U$. Note that $t \leqslant\left|S \cdot U^{-1}\right| \leqslant t+n-2$ and $\sigma\left(S \cdot U^{-1}\right)=0$. It follows from $[t, t+n] \subset C_{0}(G) \cup\{\eta(G)\}$ that $S \cdot U^{-1}$ contains a short zero-sum subsequence, which is a contradiction with $S \cdot U^{-1} \mid T$.

Proposition 39 just asserts that $C_{0}(G)$ can't contain any interval of length more than $\exp (G)$. Proposition 19 shows that $C_{0}\left(C_{n}^{r}\right)$ could not contain integers much smaller than $\eta\left(C_{n}^{r}\right)-1$. So, it seems plausible to suggest 
Conjecture 40. Let $G \neq C_{2} \oplus C_{2 m}, m \in \mathbb{N}$ be a non-cyclic finite abelian group. Then $C_{0}(G) \subset[\eta(G)-(\exp (G)+1), \eta(G)-1]$.

Conjecture 40 and Conjecture 1 suggest the following

Conjecture 41. Let $G \neq C_{2} \oplus C_{2 m}, m \in \mathbb{N}$ be a non-cyclic finite abelian group. Then $1 \leqslant\left|C_{0}(G)\right| \leqslant \exp (G)$.

Conjecture 42. $C_{0}(G)=\left[\min \left\{C_{0}(G)\right\}, \max \left\{C_{0}(G)\right\}\right]$.

The following notation concerning the inverse problem on $\mathbf{s}(G)$ was introduced in [10]. Property D: We say the group $C_{n}^{r}$ has property $D$ if $\mathbf{s}\left(C_{n}^{r}\right)=c(n-1)+1$ for some positive integer $c$, and every sequence $S$ over $C_{n}^{r}$ of length $|S|=c(n-1)$ which contains no zero-sum subsequence of length $n$ has the form $S=\prod_{i=1}^{c} g_{i}^{n-1}$ where $g_{1}, \ldots, g_{c}$ are pairwise distinct elements of $C_{n}^{r}$.

Conjecture 43. ([10], Conjecture 7.2) Every group $C_{n}^{r}$ has Property D.

It has been proved in ([10], Section 7) that Conjecture 43, if true would imply

Conjecture 44. Every group $C_{n}^{r}$ has Property $C$.

Suppose that Conjecture 44 holds true for all groups of the form $C_{n}^{r}$. For fixed $n, r \in \mathbb{N}$ and any $a \in \mathbb{N}$ we have that $\eta\left(C_{n^{a}}^{r}\right)=c\left(n^{a}, r\right)(n-1)+1$, where $c\left(n^{a}, r\right) \in \mathbb{N}$ depends on $n^{a}$ and $r$. By Lemma 12 we obtain that the sequence $\left\{c\left(n^{a}, r\right)\right\}_{a=1}^{\infty}$ is decreasing. Therefore, $c\left(n^{a}, r\right) \leqslant n^{a}$ for all sufficiently large $a$. Hence, by Lemma 16 we infer that $\eta\left(C_{n^{a}}^{r}\right)-1 \in C_{0}\left(C_{n^{a}}^{r}\right)$ for all sufficiently large $a \in \mathbb{N}$.

\section{Acknowledgments}

The authors would like to thank the referee for his/her very useful suggestions. This work has been supported by the PCSIRT Project of the Ministry of Science and Technology, the National Science Foundation of China with grant no. 10971108 and 11001035, and the Fundamental Research Funds for the Central Universities.

\section{References}

[1] G. Bhowmik and J.C. Schlage-Puchta, Davenport' s constant for groups of the form $\mathbb{Z}_{3} \oplus \mathbb{Z}_{3} \oplus \mathbb{Z}_{3 d}$, Additive Combinatorics 43 (2007), 307-326.

[2] R. Chi, S.Y. Ding, W.D. Gao, A. Geroldinger and W.A. Schmid, On zero-sum subsequences of restricted size IV, Acta Math. Hungar. 107 (2005), 337-344.

[3] B.L. Davis, D. Maclagan and R. Vakil, The card game set, Math. Intelligencer 25 (2003), 33-40.

[4] Y. Edel, Sequences in abelian groups $\mathrm{G}$ of odd order without zero-sum subsequences of length $\exp (\mathrm{G})$, Des. Codes Cryptogr. 47 (2008), 125-134. 
[5] Y. Edel, C. Elsholtz, A. Geroldinger, S. Kubertin and L. Rackham, Zero-sum problems in finite abelian groups and affine caps, Q. J. Math. 58 (2007), 159-186.

[6] C. Elsholtz, Lower bounds for multidimentional zero sums, Combinatorica 24 (2004), 351-358.

[7] P. van Emde Boas and D. Kruyswijk, A combinatorial problem on finite abelian groups, II. Math. Centre Report ZW-1969-008.

[8] Y.S. Fan, W.D. Gao and Q.H. Zhong, On the Erdos-Ginzburg-Ziv constant of finite abelian groups of high rank, J. Number Theory 131 (2011), 1864-1874.

[9] W.D. Gao, On a combinatorial problem connected with factorizations, Colloq. Math. 72 (1997), 251-268.

[10] W.D. Gao and A. Geroldinger, Zero-sum problems in finite abelian groups : a survey, Expo. Math. 24 (2006), 337-369.

[11] W.D. Gao, Q.H. Hou, W.A. Schmid and R. Thangadurai, On short zero-sum subsequences II, Integers 7 (2007), A21.

[12] W.D. Gao, A. Geroldinger and W.A. Schmid, Inverse zero-sum problems, Acta Arith. 128 (2007), 245-279.

[13] A. Geroldinger and F. Halter-Koch, Non-Unique Factorizations. Algebraic, Combinatorial and Analytic Theory, Pure and Applied Mathematics, vol. 278, Chapman \& Hall/CRC, (2006).

[14] A. Geroldinger, Additive group theory and non-unique factorizations, Combinatorial Number Theory and Additive Group Theory (2009), 1-86.

[15] A. Geroldinger, D.J. Grynkiewicz and W.A. Schmid, Zero-sum problems with congruence conditions, Acta Math. Hungar. 131 (2011), 323-345.

[16] A. Geroldinger and I.Z. Ruzsa, Combinatorial number theory and additive group theory, Birkhauser (2009).

[17] H. Harborth, Ein Extremalproblem für Gitterpunkte, J. Reine Angew. Math. 262 (1973), 356-360.

[18] H. Harborth, Ein Extremalproblem für Gitterpunkte, Ph.D. Thesis, Technische Univesität Braunschweig (1982).

[19] A. Kemnitz, On a lattice point problem, Ars Combin. 16b (1983), 151-160.

[20] J.E. Olson, A combinatorial problem on finite abelian groups, I; II, J. Number Theory 1 (1969), 8-10; 195-199. 